Artigo Original

Original Article

Patricia Teixeira Menniti Pennini ${ }^{1}$ (1) Katia de Almeida ${ }^{1}$ (B)

Descritores

Auxiliares de Audição

Questionários

Adulto

Perda Auditiva

Avaliação de Resultados

Keywords

Hearing Aids

Questionnaires

Adult

Hearing Loss

Outcomes

Endereço para correspondência Patricia Teixeira Menniti Pennini Alameda dos Arapanés, 1252, Moema - São Paulo (SP), Brasil, CEP: 04524-002.

E-mail: patriciapennini@hotmail.com

Recebido em: Agosto 21, 2019

\section{Speech, Spatial and Qualities of hearing scale na avaliação do benefício em usuários de prótese auditiva}

\author{
Speech, Spatial and Qualities of Hearing Scale \\ in assessing the benefit in hearing aid users
}

\section{RESUMO}

Objetivo: foi verificar o grau de confiabilidade do Speech, Spatial and Qualities of Hearing Scale (SSQ), na avaliação do benefício em usuários de próteses auditivas. Método: Participaram do estudo 30 adultos deficientes auditivos, candidatos ao uso (novatos) ou usuários em fase de troca de suas próteses auditivas (experientes). Todos os participantes realizaram avaliação audiológica completa, seleção e adaptação das próteses auditivas que incluiu a verificação com medidas com microfone sonda e avaliação do benefício do uso das próteses auditivas utilizando o questionário Speech, Spatial and Qualities of Hearing Scale (SSQ). Inicialmente, foi aplicado o questionário SSQ Base, em forma de entrevista. Após quatro semanas de uso das próteses auditivas novas reaplicou-se o questionário SSQ, em sua versão B (para os novatos) e C (para os experientes). Resultados: Foi identificada maior dificuldade no domínio Audição para fala, do que nos outros domínios Audição espacial e Qualidades da audição. Todas as questões foram de fácil compreensão para a maioria dos participantes, que relataram serem compatíveis com as situações do seu cotidiano. A análise estatística revelou alto coeficiente Alpha de Cronbach $(>0,9)$, demonstrando boa consistência interna entre os diversos itens do questionário. Demonstrou ser uma valiosa ferramenta para avaliar subjetivamente o desempenho comunicativo com e sem próteses auditivas. Conclusão: O SSQ mostrou ser um instrumento de fácil aplicação e com alta confiabilidade que permite avaliação do benefício em indivíduos que se encontram em processo de reabilitação auditiva e pode ser aplicado a indivíduos que utilizam diferentes tipos de próteses auditivas.

Trabalho realizado na Faculdade de Ciências Médicas da Santa Casa de São Paulo - Mestrado Profissional em Saúde da Comunicação Humana - São Paulo (SP), Brasil.

${ }^{1}$ Programa de Mestrado Profissional em Saúde da Comunicação Humana, Faculdade de Ciências Médicas da Santa Casa de São Paulo - FCMSCSP - São Paulo (SP), Brasil.

Fonte de financiamento: nada a declarar

Conflito de interesses: nada a declarar. 


\section{INTRODUÇÃO}

Com o crescente envelhecimento da população, uma das alterações sensoriais mais relevantes em idosos é a perda auditiva. Mais de 5\% da população mundial apresenta perda auditiva incapacitante. Aproximadamente, um terço das pessoas com mais de 65 anos de idade são afetadas. Os efeitos e impactos negativos da deficiência auditiva trazem limitações à pessoa, sendo uma das queixas mais prevalentes, a falta de compreensão de fala em vários ambientes acústicos, principalmente, na presença de ruído ${ }^{(1)}$.

A reabilitação é um conjunto de medidas que ajudam pessoas com deficiências a terem e manterem uma funcionalidade ideal na interação com o ambiente. É considerada eficiente quando reduz as dificuldades de comunicação do indivíduo, isto é, as incapacidades auditivas; aumenta o bem-estar psicossocial, reduz as restrições de participação, e quando as melhoras funcionais permanecem ao longo do tempo ${ }^{(2)}$.

A atuação dos profissionais na reabilitação auditiva visa minimizar as dificuldades de comunicação experimentadas por seus pacientes deficientes auditivos. A prótese auditiva, como também o implante coclear, são recursos de extrema importância, muito utilizados e continuamente aperfeiçoados ao longo dos anos, minimizando as dificuldades para se comunicar e manter um convívio social. A adaptação e o uso efetivo das próteses auditivas são fundamentais para que o processo de reabilitação do deficiente auditivo seja estabelecido. É importante avaliar o benefício proporcionado pelo uso da prótese auditiva e seus efeitos positivos na vida diária do indivíduo como medida do resultado do tratamento, ou seja, a adequação da adaptação no processo de seleção das próteses auditivas.

Entende-se por benefício à diferença de desempenho do indivíduo entre duas condições: sem e com o dispositivo eletrônico e pode ser avaliado de forma objetiva ou subjetiva. Métodos objetivos empregam medidas de limiares ou supra liminares em campo livre, de inteligibilidade de fala e de restauração da sensação de intensidade enquanto as avaliações subjetivas utilizam a autoavaliação, isto é, avaliam a opinião do usuário da amplificação não havendo referência externa para comparação(3).

É de suma importância a realização de medidas subjetivas que envolvem a autopercepção do indivíduo relacionada às suas dificuldades auditivas na vida cotidiana. Muitos questionários foram desenvolvidos para melhor caracterizar o grau e a incapacidade que resulta da deficiência auditiva e informar mais especificamente as situações enfrentadas pelos ouvintes. Há questionários que medem a satisfação do usuário com a amplificação, outros que quantificam o benefício proporcionado pelo uso das próteses auditivas e ainda aqueles que avaliam aspectos mais gerais como qualidade de vida. Entretanto, há um número limitado de questionários que abrangem o domínio de situações que dependem das capacidades auditivas binaurais. A audição binaural, possibilita a localização do som, a eliminação do efeito sombra da cabeça, propiciando melhor compreensão de fala no ruído e em diferentes ambientes, dando a impressão de um som tridimensional.

O Speech, Spatial and Qualities of Hearing Scale - SSQ foi desenvolvido com o objetivo de caracterizar a relação da deficiência e os prejuízos da experiência auditiva em uma variedade de situações complexas de escuta do cotidiano. É um instrumento de autoavaliação do indivíduo em uma variedade de domínios como situações de audição direcional relacionada a diferentes distâncias e ao movimento, segregação de sons e fluxos de vozes simultâneas, facilidade de escuta, naturalidade e clareza dos sons do cotidiano e de diferentes peças musicais e instrumentos ${ }^{(4,5)}$. O SSQ é um questionário composto por 49 questões, divididas em três dimensões: Audição para fala, Audição espacial e Qualidades da audição. Diferentes versões desse questionário foram desenvolvidas, dentre essas destacamos o SSQ - B e o SSQ - C cujo objetivo é avaliar o benefício do usuário de dispositivos eletrônicos. Ambos contêm as mesmas questões do questionário base, mas com orientações e respostas diferentes para os usuários da amplificação. A versão "benefício" ou SSQ - B é destinada a usuários novatos enquanto a versão "comparativa" ou SSQ - C destinam-se aos usuários experientes após a troca por novas próteses auditivas. Pode ser utilizado para a comparação de duas próteses diferentes, isto é, a prótese auditiva atual versus a prótese auditiva utilizada anteriormente ${ }^{(6)}$.

Em 2015, o questionário SSQ foi traduzido e adaptado para o Português Brasileiro( ${ }^{(7)}$ e está sendo utilizado como um instrumento de avaliação subjetiva das dificuldades auditivas em variedade de domínios de situações de escuta. Entretanto, ainda não foi aplicado para a avaliação do benefício em indivíduos usuários de prótese auditiva.

Assim sendo, teria o questionário SSQ em suas versões Benefício e Comparativa aplicabilidade para a avaliação do benefício em usuários de próteses auditivas?

O objetivo do estudo foi verificar o grau de confiabilidade do Speech, Spatial and Qualities of Hearing Scale (SSQ), traduzido para o Português Brasileiro, na avaliação do benefício em usuários de próteses auditivas.

\section{MÉTODO}

Todas as etapas do estudo foram realizadas na Faculdade de Ciências Médicas da Santa Casa de São Paulo - FCMSCSPSão Paulo (SP), Brasil e na Clínica Particular - São Paulo (SP), Brasil. O estudo foi aprovado pelo Comite de Ética em Pesquisa sob o $\mathrm{n}^{\circ}$ 1.613.072. Todos os participantes assinaram o TCLE, autorizando a sua participação no estudo.

Participaram 30 indivíduos adultos com deficiência auditiva, sendo que 22 eram usuários experientes de próteses auditivas e oito novatos ao uso, com idades entre 44 e 94 anos, de ambos os sexos (13 mulheres e 17 homens) e escolaridade predominantemente, de nível superior.

A seleção dos indivíduos do estudo foi baseada com os seguintes critérios de elegibilidade: indivíduos adultos; com perda auditiva de qualquer tipo ou grau de severidade; ser candidato ao uso ou usuários em fase de troca de suas próteses auditivas. E os critérios de exclusão foram: indivíduos que tivessem problemas de saúde que os impedissem da participação em todas as avaliações e procedimentos propostos para a pesquisa; apresentassem alterações cognitivas perceptíveis, ou outras alterações neurológicas; e caso não conseguissem fazer o uso adequado das próteses auditivas no período proposto, por intercorrências diversas. 
Foram selecionados, aleatoriamente, pacientes que compareciam à clínica para o processo de adaptação inicial ou que estavam trocando suas próteses auditivas.

Inicialmente, foram obtidas informações gerais e relevantes da história prévia do indivíduo, com relação à queixa auditiva, a etiologia provável, o tempo da perda, sobre saúde geral que pudessem interferir no processo. Quando necessário, foram confeccionados moldes ou cápsulas para adaptação física das próteses auditivas intra-aurais.

A prescrição do ganho foi realizada de acordo com os métodos prescritivos validados como NAL NL1 - National Acoustic Laboratories Non-Linear ${ }^{(8)}$ e DSL-v5 - Desired Sensation Level ${ }^{(9)}$ para usuários experientes; e NAL-NL2 National Acoustic Laboratories Non-Linear ${ }^{(10)}$ para todos os novatos ao uso da prótese auditiva. A programação e ajuste fino das próteses auditivas foi feita por meio de softwares das empresas fabricantes na plataforma NOAH.

A verificação do desempenho das próteses auditivas foi realizada por meio de equipamentos com microfone sonda, em sala acusticamente tratada, com o paciente sentado a um metro de distância do alto-falante posicionado em $0^{\circ}$ azimute, usando a ferramenta Mapeamento de fala amplificada com os algoritmos acionados. Essa ferramenta permite a avaliação do funcionamento eletroacústico das próteses auditivas selecionadas e verificar se os valores de ganho e saída gerados estavam de acordo com os prescritos. Utilizou-se um sinal de fala ISTS $^{(11)}$ em diferentes intensidades de entrada 55, 65, $75 \mathrm{~dB}$ NPS.

Nesse momento foram também anotados os valores do Índice de Inteligibilidade de Fala (Speech Inteligibility Índex) - SII ${ }^{(12)}$ para a intensidade de $65 \mathrm{~dB}$ NPS, denominado SII 65. A prótese auditiva que propiciasse maior SII era usada em um período de experiência domiciliar, sempre orientando o uso binaural quando da perda bilateral.

Tanto para o usuário novo como para o experiente foi permitido o teste domiciliar por quatro semanas, com retornos para ajustes, para retomar os orientações e aconselhamentos. Foram registrados por meio do datalogging as horas de uso diário das próteses auditivas.

O questionário Speech, Spatial and Qualities of Hearing Scale (SSQ) - versão 5.6 ${ }^{(5)}$ traduzido e validado para o Português Brasileiro $^{(7)}$ tem como finalidade a autoavaliação, em uma variedade de domínios como: situações de audição direcional relacionada a diferentes distâncias e ao movimento, segregação de sons e fluxos de vozes simultâneas, facilidade de escuta, naturalidade e clareza dos sons do cotidiano e de diferentes peças musicais e instrumentos. O questionário SSQ - Base, foi aplicado inicialmente em todos os indivíduos, apresentando oralmente cada questão em forma de entrevista, e os participantes, baseados em sua vivência sem próteses auditivas ou de acordo com sua experiência com as próteses auditivas antigas, pontuaram de 0 a 10 o seu desempenho comunicativo. Todos eram informados que, na escala de respostas, o 10 significa que são perfeitamente capazes de executar o descrito na questão e o 0 (zero), que são incapazes de realizar a situação investigada. Além disto, existe a opção denominada "não aplicável", nos casos em que a pergunta não se referia a sua situação cotidiana (Figura 1). O tempo médio de aplicação do questionário foi de 30 minutos.

Após quatro semanas de uso das próteses auditivas, foram aplicados novamente os questionários de autoavaliação, mas para os novatos, foi aplicado o questionário SSQ- B (Benefício) e para os experientes, o questionário SSQ - C (Comparativo). $\mathrm{O}$ que diferencia as versões Base, B e C são as instruções dadas e formulários de respostas.

O questionário SSQ - B que foi aplicado para novos usuários de próteses auditivas, tem como objetivo a avaliação do benefício a partir da comparação das respostas sem e com próteses auditivas. $\mathrm{O}$ questionário SSQ - C destina-se para antigos usuários de próteses auditivas em fase de troca. Contém as mesmas questões, mas com as mesmas possibilidades de resposta do SSQ - B. Cada questão foi apresentada oralmente e os participantes tiveram que pontuar em uma escala de pontuação de $-5 \mathrm{a}+5$ o seu desempenho comunicativo com suas novas próteses auditivas. Sendo que foram informados de que, na escala de respostas, o +5 significa que são perfeitamente capazes de executar o que está descrito na questão, e o - 5 que seriam incapazes de realizar a situação investigada. Além disto, existe a opção denominada "não aplicável", nos casos em que a pergunta se referia a uma situação cotidiana ainda não experienciada (Figura 2).

O benefício foi indicado pela diferença entre as dificuldades observadas pré e pós a intervenção, indicando o grau de melhoria com o uso da prótese auditiva.

Inicialmente, foi realizada uma análise descritiva para variáveis qualitativas com frequências, valor absoluto $(\mathrm{N})$ e valor relativo (\%), que são: sexo, escolaridade, grau da perda. Para as variáveis

De modo algum

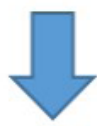

Perfeitamente

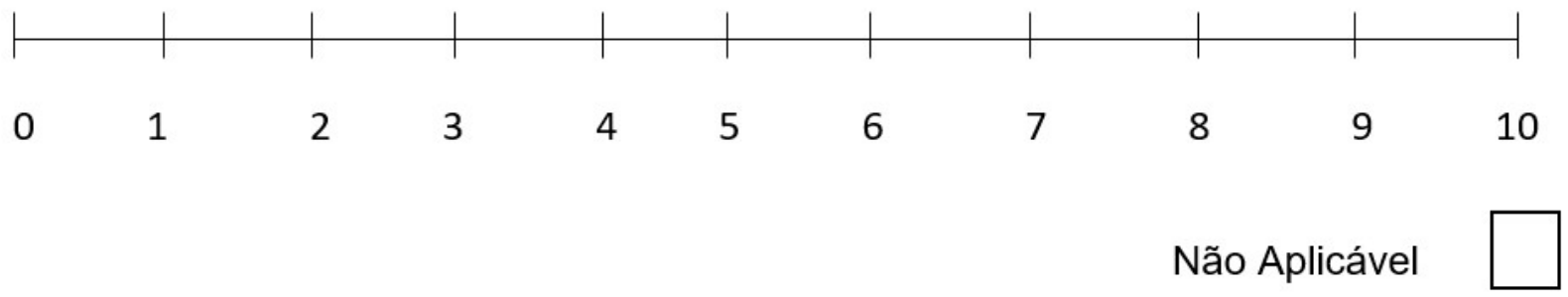

Figura 1. Escala de respostas SSQ-BASE 
quantitativas foram calculadas as medidas de resumo (média, mediana, desvio-padrão, mínimo e máximo): idade, questionários SSQ Base, B e C. A confiabilidade dos questionários B e C foi estimada utilizando-se a análise de consistência interna Alfa de Cronbach. O coeficiente Alfa $(\alpha)$ mede a correlação entre respostas em um questionário, por meio da análise do perfil das respostas dadas pelos respondentes. A análise estatística dos resultados foi feita por meio do programa SPSS (13.0).

\section{RESULTADOS}

Realizamos o estudo com 30 adultos deficientes auditivos, sendo que 22 eram usuários experientes de próteses auditivas e oito novatos ao uso, com idades entre 44 e 94 anos, de ambos os sexos (13 mulheres e 17 homens), com escolaridade, predominantemente, de nível superior. A média do tempo de privação auditiva dos indivíduos da amostra foi de 9 anos, os limiares auditivos desta amostra mostraram-se homogêneos e sem diferenças estatisticamente significantes entre orelhas direitas (média de 59,9 dB) e esquerdas (média de $58 \mathrm{~dB}$ ). Após período de teste de quatro semanas, foi registrado e analisado o número de horas de uso diário, por meio do datalogging das próteses auditivas, que variou de 4 a 18 horas por dia (Tabela $1 \mathrm{e}$ Figura 3).

A seguir apresentamos as respostas dos participantes antes e depois do uso das novas próteses auditivas, por meio do questionário Speech, Spatial and Qualities of Hearing ScaleSSQ Base, B e C, representadas pelas médias, desvio padrão, pontuações mínimas e máximas dos itens em português por domínio e total (Tabelas 2 e 3 ).

\section{Muito pior}

sem alteração

Muito melhor

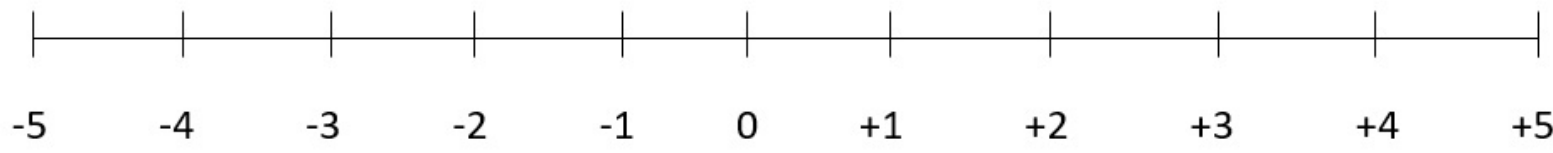

Não Aplicável

Figura 2. Escala de respostas $S S Q-B$ e C

Tabela 1. Caracterização da amostra dos indivíduos que participaram do estudo

\begin{tabular}{|c|c|c|c|}
\hline Características & Categorias & $\mathrm{n}$ & $\%$ \\
\hline \multirow[t]{3}{*}{ Idade } & $44-63$ & 10 & 33,3 \\
\hline & $66-77$ & 11 & 36,7 \\
\hline & $78-94$ & 9 & 30,0 \\
\hline \multirow[t]{2}{*}{ Sexo } & Feminino & 13 & 43,3 \\
\hline & Masculinos & 17 & 56,7 \\
\hline \multirow[t]{4}{*}{ Escolaridade } & Primária & 2 & 6,7 \\
\hline & Fundamental & 1 & 3,3 \\
\hline & Médio & 5 & 16,7 \\
\hline & Superior & 22 & 73,3 \\
\hline \multirow[t]{2}{*}{ Usuários de próteses auditivas bilateral } & Novatos & 8 & 26,7 \\
\hline & Experientes & 22 & 73,3 \\
\hline \multirow[t]{4}{*}{ Perda auditiva (média de 0,5,1 e $2 \mathrm{KHz}$ na melhor orelha) } & Leve & 6 & 20,7 \\
\hline & Moderada & 15 & 51,7 \\
\hline & Severa & 6 & 20,7 \\
\hline & Profunda & 2 & 6,9 \\
\hline \multirow[t]{4}{*}{ Modelo de próteses auditivas } & Retro & 2 & 6,7 \\
\hline & Mini canal & 4 & 13,3 \\
\hline & Intra canal & 1 & 3,3 \\
\hline & REC & 23 & 76,7 \\
\hline \multirow[t]{3}{*}{ Tempo de uso diário (com AASI) } & $4-9$ hs & 12 & 40,0 \\
\hline & $10-14$ hs & 10 & 33,3 \\
\hline & $15-18$ hs & 8 & 26,7 \\
\hline
\end{tabular}


Tabela 2. Valores de Média, Mediana, DP, mínimos e máximos das respostas do questionário SSQ Base no domínio da audição para fala, audição espacial e qualidades da audição

\begin{tabular}{|c|c|c|c|c|c|}
\hline$S S Q-$ Base & Questão & Média & DP & Min & Max \\
\hline \multirow{14}{*}{$\begin{array}{l}\text { AUDIÇÃO PARA } \\
\text { FALA }\end{array}$} & 1.Conversando com alguém com a TV ligada & 5,77 & 1,69 & 3 & 10 \\
\hline & 2.Conversando com alguém em uma sala silenciosa e com tapete & 8,63 & 1,45 & 5 & 10 \\
\hline & 3.Conversando com 5 pessoas no silêncio com visão & 5,90 & 2,24 & 2 & 10 \\
\hline & 4.Conversando com 5 pessoas no ruído com visão & 3,90 & 2,02 & 0 & 8 \\
\hline & 5.Conversando com alguém com ruído contínuo & 6,37 & 1,54 & 4 & 10 \\
\hline & 6.Conversando com 5 pessoas no ruído sem visão & 3,30 & 1,66 & 0 & 7 \\
\hline & 7.Conversando em um ambiente com eco & 4,60 & 2,37 & 0 & 10 \\
\hline & 8.Ignorar a voz interferente de mesmo tom & 5,30 & 2,16 & 2 & 10 \\
\hline & 9.Ignorar a voz interferente de diferente tom & 5,53 & 2,06 & 2 & 10 \\
\hline & 10.Conversar com alguém e seguir a TV & 3,53 & 2,19 & 0 & 8 \\
\hline & $\begin{array}{l}\text { 11.Conversar com alguém em uma sala com outras pessoas } \\
\text { conversando }\end{array}$ & 5 & 2,01 & 1 & 10 \\
\hline & 12.Seguir a mudança de conversação em um grupo & 3,90 & 1,96 & 0 & 7 \\
\hline & 13.Ter uma conversação no telefone & 6,30 & 2,50 & 0 & 10 \\
\hline & 14.Acompanhar uma pessoa falando e o telefone & 3,73 & 2,33 & 0 & 10 \\
\hline Total & & 5,12 & 1,43 & 3,2 & 9,1 \\
\hline \multirow{17}{*}{ AUDIÇÃO ESPACIAL } & 1.Localizar o cortador de grama & 4,50 & 2,90 & 0 & 10 \\
\hline & 2.Localizar alguém falando em volta de uma mesa & 4,80 & 2,70 & 0 & 10 \\
\hline & 3.Localizar o falante da direita ou da esquerda & 7,40 & 2,20 & 2 & 10 \\
\hline & 4.Localizar uma porta bater em uma casa desconhecida & 5,40 & 3,30 & 0 & 10 \\
\hline & 5.Localizar o som acima ou embaixo em uma escada & 5,30 & 2,30 & 0 & 9 \\
\hline & 6.Localizar o latido de um cachorro & 5,40 & 2,70 & 0 & 10 \\
\hline & 7.Localizar a direção de um veículo & 5,50 & 2,80 & 0 & 10 \\
\hline & 8.Julgar a distância de alguém pelos passos ou vozes & 4,20 & 2,20 & 0 & 8 \\
\hline & 9.Julgar a distância do veículo & 4,80 & 2,20 & 0 & 10 \\
\hline & 10.Identificar a lateralidade do movimento do veículo & 4,80 & 2,90 & 0 & 10 \\
\hline & $\begin{array}{l}\text { 11. Identificar a lateralidade do movimento de alguém pelos passos } \\
\text { ou vozes }\end{array}$ & 4,80 & 2,60 & 0 & 10 \\
\hline & 12. Identificar a proximidade ou afastamento de uma pessoa & 5,40 & 2,30 & 0 & 10 \\
\hline & 13.Identificar a proximidade ou afastamento de um veículo & 5,90 & 2,50 & 0 & 10 \\
\hline & 14.Sons não externalizados (dentro da cabeça mais do que lá fora) & 6,70 & 2,00 & 2 & 10 \\
\hline & 15.Sons mais perto do que o esperado & 5,70 & 1,90 & 2 & 10 \\
\hline & 16.Sons mais longe do que o esperado & 4,90 & 2,30 & 0 & 10 \\
\hline & 17.Sons na localização esperada & 6,40 & 2,40 & 0 & 10 \\
\hline Total & & 5,40 & 1,86 & 2,10 & 10 \\
\hline \multirow{18}{*}{$\begin{array}{l}\text { QUALIDADE DA } \\
\text { AUDIÇÃO }\end{array}$} & 1.Separação de dois sons & 6,80 & 2,60 & 0 & 10 \\
\hline & 2.Sons parecem misturados & 6,80 & 1,90 & 3 & 10 \\
\hline & 3.Ouvir a voz e música como sons separados & 5,60 & 2,40 & 0 & 10 \\
\hline & 4.Identificar pessoas conhecidas pela voz & 7,50 & 1,90 & 4 & 10 \\
\hline & 5.Distinguir música conhecida & 7,20 & 2,40 & 0 & 10 \\
\hline & 6.Distinguir sons diferentes & 7,00 & 2,30 & 2 & 10 \\
\hline & 7.Identificar os instrumentos musicais em uma música & 5,70 & 2,50 & 0 & 10 \\
\hline & 8.Naturalidade da música & 6,50 & 3 & 0 & 10 \\
\hline & 9.Claridade dos sons do dia & 7,00 & 1,80 & 3 & 10 \\
\hline & 10.Naturalidade das vozes & 6,30 & 1,70 & 2 & 10 \\
\hline & 11.Naturalidade dos sons do dia & 6,50 & 2,10 & 2 & 10 \\
\hline & 12.Naturalidade da própria voz & 7,20 & 2 & 2 & 10 \\
\hline & 13.Avaliando o humor da voz & 7,20 & 2,20 & 0 & 10 \\
\hline & 14.Precisa se concentrar quando escuta & 5,20 & 2,60 & 0 & 10 \\
\hline & 15.Esforço na conversação & 4,90 & 2,40 & 0 & 10 \\
\hline & 16. Dirigindo consegue ouvir bem o passageiro & 5,40 & 2,60 & 0 & 10 \\
\hline & 17.Como passageiro consegue ouvir o motorista & 6,00 & 2,20 & 2 & 10 \\
\hline & 18.Capacidade de ignorar sons concorrentes & 5,80 & 2,70 & 1 & 10 \\
\hline Total & & 6,37 & 1,35 & 3,90 & 9,90 \\
\hline
\end{tabular}


Tabela 3. Respostas do questionário SSQ B e C no domínio da audição para fala, audição espacial e qualidades da audição

\begin{tabular}{|c|c|c|c|c|c|}
\hline$S S Q-B$ e $C$ & Questão & Média & $\mathrm{DP}$ & Min & Max \\
\hline \multirow[t]{14}{*}{ AUDIÇÃO PARA FALA } & 1. Conversando com alguém com a TV ligada & 2,63 & 1,45 & 0 & 5 \\
\hline & 2. Conversando com alguém em uma sala silenciosa e com tapete & 3,43 & 1,77 & 0 & 5 \\
\hline & 3. Conversando com 5 pessoas no silêncio com visão & 2,45 & 1,54 & 0 & 5 \\
\hline & 4. Conversando com 5 pessoas no ruído com visão & 1,60 & 1,38 & 0 & 4 \\
\hline & 5. Conversando com alguém com ruído contínuo & 2,79 & 1,49 & 0 & 5 \\
\hline & 6. Conversando com 5 pessoas no ruído sem visão & 1,63 & 1,40 & 0 & 4 \\
\hline & 7. Conversando em um ambiente com eco & 1,79 & 1,42 & 0 & 5 \\
\hline & 8. Ignorar a voz interferente de mesmo tom & 2,28 & 1,43 & 0 & 5 \\
\hline & 9. Ignorar a voz interferente de diferente tom & 2,50 & 1,38 & 0 & 5 \\
\hline & 10. Conversar com alguém e seguir a TV & 1,40 & 1,49 & 0 & 5 \\
\hline & 11. Conversar com alguém em uma sala com outras pessoas conversando & 2,40 & 1,42 & 0 & 5 \\
\hline & 12. Seguir a mudança de conversação em um grupo & 1,97 & 1,35 & 0 & 4 \\
\hline & 13. Ter uma conversação no telefone & 2,47 & 1,79 & 0 & 5 \\
\hline & 14. Acompanhar uma pessoa falando e o telefone & 1,37 & 1,52 & 0 & 4 \\
\hline Total & & 2,20 & 2,03 & 0 & 4,4 \\
\hline \multirow[t]{17}{*}{ AUDIÇÃO ESPACIAL } & 1. Localizar o cortador de grama & 2,25 & 1,66 & 0 & 5 \\
\hline & 2. Localizar alguém falando em volta de uma mesa & 2,21 & 1,59 & 0 & 5 \\
\hline & 3. Localizar o falante da direita ou da esquerda & 3,00 & 1,89 & 0 & 5 \\
\hline & 4. Localizar uma porta bater em uma casa desconhecida & 2,21 & 1,83 & 0 & 5 \\
\hline & 5. Localizar o som acima ou embaixo em uma escada & 2,20 & 1,80 & 0 & 5 \\
\hline & 6. Localizar o latido de um cachorro & 2,21 & 1,65 & 0 & 5 \\
\hline & 7. Localizar a direção de um veículo & 2,39 & 1,59 & 0 & 5 \\
\hline & 8. Julgar a distância de alguém pelos passos ou vozes & 1,97 & 1,34 & 0 & 5 \\
\hline & 9. Julgar a distância do veículo & 2,07 & 1,58 & 0 & 5 \\
\hline & 10. Identificar a lateralidade do movimento do veículo & 2,07 & 1,54 & 0 & 5 \\
\hline & 11. Identificar a lateralidade do movimento de alguém pelos passos ou vozes & 2,27 & 1,48 & 0 & 5 \\
\hline & 12. Identificar a proximidade ou afastamento de uma pessoa & 2,41 & 1,42 & 0 & 5 \\
\hline & 13.. Identificar a proximidade ou afastamento de um veículo & 2,17 & 1,44 & 0 & 5 \\
\hline & 14. Sons não externalizados (dentro da cabeça) mais do que fora & 2,37 & 1,86 & 0 & 5 \\
\hline & 15. Sons mais perto do que o esperado & 2,33 & 1,46 & 0 & 5 \\
\hline & 16. Sons mais longe do que o esperado & 2,18 & 1,76 & 0 & 5 \\
\hline & 17. Sons na localização esperada & 2,47 & 1,81 & 0 & 5 \\
\hline Total & & 2,25 & 2,03 & 0 & 5 \\
\hline \multirow[t]{18}{*}{ QUALIDADES DA AUDIÇÃO } & 1. Separação de dois sons & 2,50 & 1,67 & 0 & 5 \\
\hline & 2. Sons parecem misturados & 2,13 & 1,69 & 0 & 5 \\
\hline & 3. Ouvir a voz e música como sons separados & 2,50 & 1,50 & 0 & 5 \\
\hline & 4. Identificar pessoas conhecidas pela voz & 2,77 & 1,69 & 0 & 5 \\
\hline & 5. Distinguir música conhecida & 2,40 & 1,97 & 0 & 5 \\
\hline & 6. Distinguir sons diferentes & 2,55 & 1,84 & 0 & 5 \\
\hline & 7. Identificar os instrumentos musicais em uma música & 2,10 & 1,70 & 0 & 5 \\
\hline & 8. Naturalidade da música & 2,50 & 2,03 & 0 & 5 \\
\hline & 9. Claridade dos sons do dia & 2,40 & 1,56 & 0 & 5 \\
\hline & 10. Naturalidade das vozes & 2,47 & 1,79 & 0 & 5 \\
\hline & 11. Naturalidade dos sons do dia & 2,27 & 1,96 & -2 & 5 \\
\hline & 12. Naturalidade da própria voz & 1,86 & 2,19 & -2 & 5 \\
\hline & 13. Avaliando o humor da voz & 2,17 & 1,82 & 0 & 5 \\
\hline & 14. Precisa se concentrar quando escuta & 2,20 & 1,62 & 0 & 5 \\
\hline & 15. Esforço na conversação & 2,37 & 1,69 & 0 & 5 \\
\hline & 16. Dirigindo consegue ouvir bem o passageiro & 2,29 & 1,48 & 0 & 5 \\
\hline & 17. Como passageiro consegue ouvir o motorista & 2,76 & 1,50 & 0 & 5 \\
\hline & 18. Capacidade de ignorar sons concorrentes & 1,93 & 1,66 & 0 & 5 \\
\hline Total & & 2,35 & 2,50 & 0 & 4,8 \\
\hline
\end{tabular}


Os índices médios dos domínios demonstraram que no domínio Audição para fala ocorreu a menor pontuação e nos domínios Audição espacial e Qualidades da audição, as maiores pontuações. Os índices médios dos domínios variaram de 2,20 a 2,35 pontos. O desvio padrão variou de 1,04 a 1,41(Tabela 4 e Figura 4).

No domínio daAudição para fala a menor pontuação foi na questão 14 e a maior pontuação na questão 2 . Os índices médios variaram de 1,37 a 3,43 pontos e o desvio padrão variou de 1,35 a 1,79.

No domínio da Audição espacial as menores pontuações ocorreram nas questões $8,9,10$ e a maior pontuação foi na questão 3. Os índices médios variaram de 2,07 a 3,0 pontos e o desvio padrão variou de 1,34 a 1,89.

No domínio das Qualidades da audição a menor pontuação foi na questão 15 e a maior pontuação na questão 4. Os índices médios variaram de 2,37 a 2,77 pontos e o desvio padrão variou de 1,48 a 2,19 .

Os 49 itens do SSQ - Base em português produziram um Alfa de Cronbach de 0,94, e o SSQ - B e C de 0,99, indicando elevada consistência interna. Os domínios também apresentaram boa confiabilidade (Tabela 5).

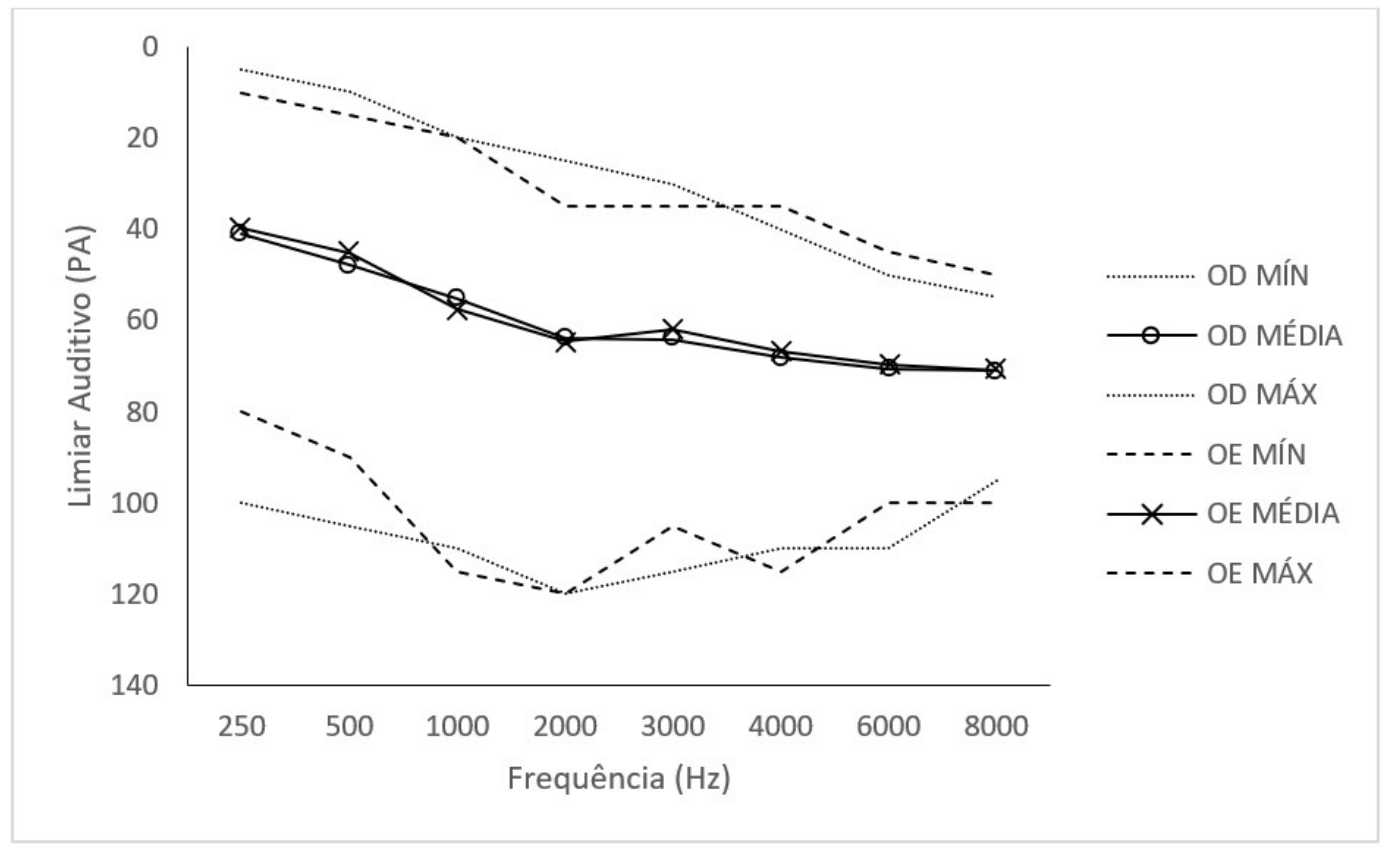

Figura 3. Distribuição do grau da perda auditiva das orelhas direita e esquerda $(\mathrm{N}=60)$

Tabela 4. Valores de Média, Mediana, DP, mínimos e máximos das respostas ao questionário SSQ Base, B e C por domínio e total

\begin{tabular}{|c|c|c|c|c|c|c|}
\hline & & Média & Mediana & $\mathrm{DP}$ & Mínimo & Máximo \\
\hline \multirow[t]{4}{*}{ SSQ Base } & Fala - base & 5,12 & 5,1 & 1,43 & 3,2 & 9,1 \\
\hline & Espacial - base & 5,36 & 5,79 & 1,86 & 2,1 & 10 \\
\hline & Qualidades - base & 6,37 & 6,38 & 1,35 & 3,9 & 9,9 \\
\hline & Total - base & 5,7 & 5,68 & 1,28 & 3,7 & 9,7 \\
\hline \multirow[t]{4}{*}{ SSQ B e C } & Fala -B e C & 2,2 & 2,03 & 1,04 & 0,2 & 4,4 \\
\hline & Espacial - B e C & 2,25 & 2,4 & 1,33 & 0,1 & 4,8 \\
\hline & Qualidades - B e C & 2,35 & 2,5 & 1,41 & 0 & 4,8 \\
\hline & Total - B e C & 2,27 & 2,34 & 1,2 & 0,1 & 4,5 \\
\hline
\end{tabular}

Tabela 5. Medidas de consistência interna das respostas por domínios e total do questionário SSQ Base, versão B e C

\begin{tabular}{cccc}
\hline & N de questões & Alpha de Cronbach \\
\hline SSQ-Base & Fala - base & 14 & 0,97 \\
& Espacial - base & 17 & 0,94 \\
& Qualidade - base & 18 & 0,88 \\
SSQ B e C & Total - base & 49 & $\mathbf{0 , 9 4}$ \\
& Fala - B e C & 14 & 0,95 \\
& Espacial B e C & 17 & 0,97 \\
& Qualidade - B e C & 18 & 0,98 \\
& Total - B e C & $\mathbf{4 9}$ & $\mathbf{0 , 9 9}$ \\
\hline
\end{tabular}


A

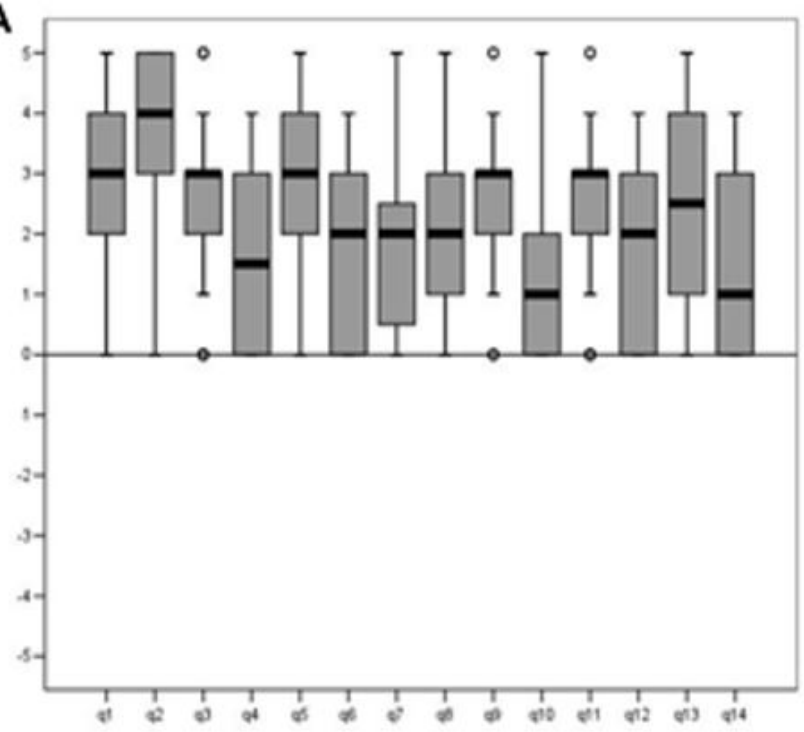

B



\section{C}

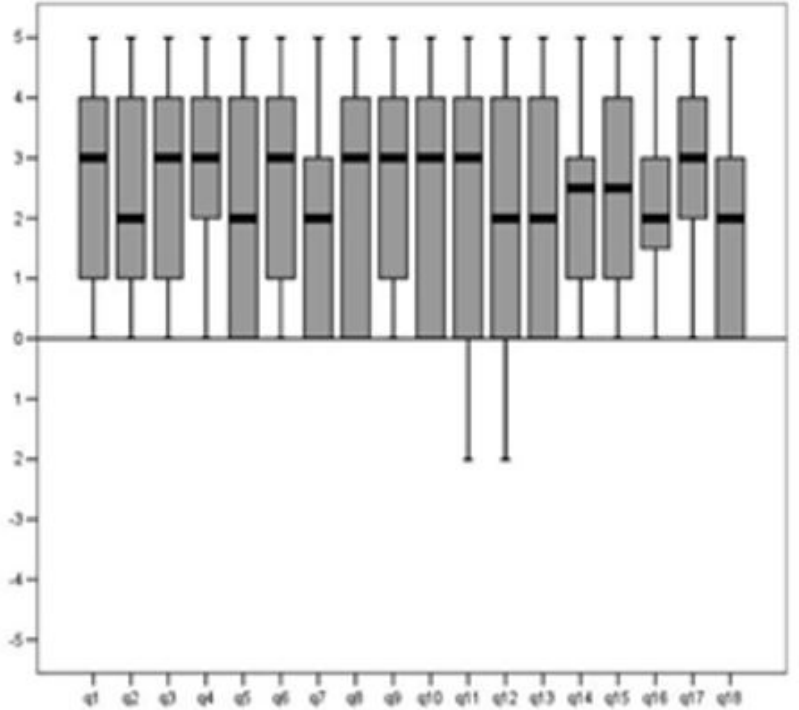

Figura 4. BoxPlot das respostas ao questionário SSQ B e C nos domínios Audição para fala (A), Audição espacial (B) e Qualidades da audição (C)

\section{DISCUSSÃO}

O benefício foi considerado como a diferença no desempenho individual, independente de experiência prévia, haja visto que, a experiência prévia não é fonte de variabilidade ${ }^{(3)}$. A diferença da autopercepção pré e pós adaptação das novas próteses auditivas, constitui um indicador do benefício, o qual pode ser avaliado por meio de procedimentos objetivos e subjetivos. No nosso estudo, utilizamos a avaliação subjetiva do benefício o questionário Speech, Spatial and Qualities of Hearing ScaleSSQ nas versões B e C, traduzidas para o Português Brasileiro ${ }^{(7)}$.

O Speech, Spatial and Qualities of Hearing Scale (SSQ) versão 5.6 é um questionário de autoavaliação no qual o indivíduo quantifica as dificuldades experimentadas nas diferentes situações de comunicação do cotidiano. É constituído de 49 questões, divididas em três domínios: Audição para fala, Audição espacial e Qualidades da audição. O primeiro domínio, Audição para fala avalia a compreensão de fala em diferentes situações do dia pé; o segundo domínio, Audição espacial investiga componentes da audição espacial, como lateralidade e localização; e o terceiro domínio, Qualidades da audição, como avaliar a capacidade de segregação de sons, fluxo de vozes simultâneas, facilidade de escuta, naturalidade e clareza dos sons do cotidiano, de diferentes peças musicais e instrumentos.

O SSQ já foi traduzido para diversos idiomas e utilizado na prática clínica, em vários países ${ }^{(13)}$, porém, no Brasil não havia sido utilizado em suas versões de avaliação do benefício em Português Brasileiro. Foi realizada a tradução, retrotradução, avaliação por comitê de especialistas e adaptação cultural do SSQ para o Português Brasileiro( ${ }^{(7)}$. Após validação linguística e 
estudo piloto em indivíduos normo-ouvintes, realizamos nesse estudo, a descrição e testes das propriedades psicométricas da versão no português Brasileiro do questionário SSQ B e C em indivíduos com deficiência auditiva e usuários de próteses auditivas, para posterior uso clínico.

Foi aplicado questionário Speech, Spatial and Qualities of Hearing Scale-SSQ Base, B e C em todos os participantes usando o método de entrevista ${ }^{(14)}$. No questionário SSQ - Base, os indivíduos pontuavam seu desempenho comunicativo e eram interpretados da seguinte maneira: quanto maior a pontuação ${ }^{(15)}$ de cada domínio e/ou questão, menor a dificuldade experimentada nas situações proposta de comunicação de vida diária deste indivíduo e quanto menor a pontuação (0), maior dificuldade. E no SSQ B e C, a variação do desempenho era com a pontuação negativa, estava pior (-5), com a neutra (0), não havia tido mudança e positiva $(+5)$, melhor desempenho.

Em um estudo inicial ${ }^{(5)}$ encontraram índices de 5,5 para idosos com perda auditiva moderada e enquanto em outro trabalho, de tradução para o português ${ }^{(7)}$ em adultos normo-ouvintes, os índices médios por domínios variaram de 5,8 a 9,4 pontos e o desvio padrão variou de 0,57 a 3,32. No estudo em questão foi obtido um índice médio do SSQ Base total de 5,70 (1,28 DP), evidenciando sensibilidade à variação e poder discriminativo entre os indivíduos. No entanto, as diferenças absolutas entre os estudos ocorreram para muitos itens, especialmente nos domínios da Audição espacial e Qualidades da audição, isso pode ser devido aos diferentes graus de perda auditiva dos indivíduos da amostra. Os índices médios do SSQ - Base foram menores no domínio da Audição para fala do que nos outros domínios, como já observados em estudos anteriores ${ }^{(5,14-17)}$.

Quando analisadas as respostas das questões do questionário SSQ - Base por cada domínio na Audição para fala, a pontuação variou de 3,30 a 8,63 (de uma pontuação máxima de 10). A situação com maior dificuldade era na conversa em grupo com ruído competitivo e sem visão dos falantes e a de maior facilidade era conversando com alguém em uma sala silenciosa e com tapete. Comparando com outro estudo ${ }^{(16)}$, as pontuações totais variaram de 2,5 a 7,1 sendo que as questões com menor e maior dificuldades são as mesmas encontradas nesse estudo.

$\mathrm{Na}$ Audição espacial, a variação da pontuação foi de 4,20 a 7,40. A questão em que o indivíduo julgava a distância de alguém pelos passos ou vozes foi a que apresentou menor pontuação e com isto maior limitação, e a situação em que o indivíduo precisava avaliar a localização do falante, se da direita ou esquerda foi a de maior pontuação. Comparando com o estudo inicial $^{(16)}$ os achados são bem semelhantes aos de nosso estudo com pontuações de 4,2 a 7,5.

As respostas do SSQ - Base no domínio das Qualidades da audição, apresentaram variação da pontuação de 4,90 a 7,50 . Houve maior dificuldade relatada quando o indivíduo precisa se esforçar muito em uma conversação e com isto maior dificuldade de acompanhar o que está sendo dito. E a de maior facilidade foi a de reconhecer vozes de pessoas conhecidas. No estudo inicial ${ }^{(16)}$, encontraram uma pontuação de 3,7 a 8,3, corroborando nossos achados.

Determinamos um período de 30 dias de uso com as novas próteses auditivas, conforme estudo $2001^{(18)}$, uma vez que o intervalo de quatro semanas pós-ajuste é provavelmente o ponto de medição mais comum clinicamente. Neste período, os indivíduos que apresentassem necessidade de ajustes nas programações das próteses auditivas, retornavam para atendimento.

Após os 30 dias, foi aplicado o questionário SSQ - B (Benefício) para os novatos e o SSQ - C (Comparativo) para os experientes, os quais, os indivíduos pontuavam sua melhora (de $+1 \mathrm{a}+5)$, piora $(-5 \mathrm{a}-1)$ ou sem mudança (0) do seu desempenho comunicativo nas variadas situações propostas pelas questões do questionário.

Os índices médios obtidos a partir da aplicação do questionário SSQ- B para os indivíduos novatos, sem uso de próteses auditivas e o SSQ-C para os experientes com uso prévio de amplificação, por domínio e geral, apresentaram as pontuações: 2,20 na Audição para fala, 2,25 na Audição espacial e 2,35 nas Qualidades da audição. E o índice médio geral do SSQ -B e C foi 2,27. A pontuação mínima foi 0 e máxima 4,80 (de $-5,0 \mathrm{a}+5)$.

Na Figura 2, representa as respostas ao questionário SSQ versão B e C por domínio e total, demonstrando a evolução das habilidades auditivas após o uso das novas próteses auditivas.

Foi realizada uma análise descritiva das respostas do questionário SSQ-B e C por domínio. Na Audição para fala, os índices médios variaram de 1,37, menor pontuação (conversar ao telefone e com alguém) a 3,43, a maior pontuação (conversar com alguém em uma sala silenciosa e com tapete). $\mathrm{O}$ valor mínimo foi 0 (sem mudança) e máxima 5 (melhora no desempenho).

As respostas do questionário SSQ B e C na Audição espacial, em que a menor pontuação média foi 1,97 (julgar distância de alguém pelos passos e vozes, a distância do veículo e identificar a lateralidade do movimento do veículo) e a maior pontuação média foi 3,0 (localizar o falante da direita e da esquerda). $\mathrm{O}$ valor mínimo foi 0 (sem alteração) e o máximo 5 (melhora no desempenho).

As análises das respostas do questionário SSQ B e C das Qualidades da audição, apresentaram índices médios de 1,86 a 2,77. Foram observadas médias positivas de SSQ-B em muitos itens, mas apresentaram índices negativos no SSQ - C. Houve um caso de um indivíduo experiente ao uso da prótese auditiva, pontuou na questão naturalidade da própria voz -2 , isto pode ser atribuído ao fato deste usuário estar bem adaptado e, portanto, acostumado com a amplificação fornecidas por suas próteses auditivas anteriores. Estava trocando de próteses auditivas e tinha como parâmetro de normalidade da sua voz com as próteses anteriores, por que sem seus aparelhos, este indivíduo é incapaz de ouvir a própria voz.

Os dados fazem sentido com relação as situações problemáticas (medidas pelas questões do SSQ Base) e o benefício das próteses auditivas nestas situações (medidos pelo SSQ - B e C) são geralmente classificados de acordo com as expectativas. AAlta classificação SSQ - B e C em questões nas quais as pontuações SSQ- Base já eram altas, indicam que o SSQ - B e C podem demonstrar um benefício mesmo quando há um efeito maior sobre o SSQ - Base.

As respostas ao questionário SSQ - B e C, apresentaram um menor desempenho para Audição para fala do que os outros domínios (Audição espacial e Qualidades da audição). Houve resposta positiva em todos os domínios, mostrando 
assim uma evolução com o uso das novas próteses auditivas. $\mathrm{O}$ baixo desempenho para fala teria influência pelo grau da perda auditiva e não pela idade cronológica ${ }^{(16)}$. A variação individual nas pontuações é bastante grande em muitos itens (não mostrada na exibição de dados).

Os participantes relataram que o questionário era adequado e refletia as dificuldades apresentadas por eles em sua vida cotidiana. Alguns, nunca haviam prestado atenção a certas situações, mesmo vivenciadas por eles. A maioria achou o questionário de fácil resposta, mas muito longo, cansativo e repetitivo em algumas questões, dificultando sua interpretação. Perguntavam mais detalhes da questão para que pudessem ser mais fiéis na resposta. Houve a sugestão de usar versões reduzidas do questionário. Na literatura encontra-se outras versões reduzidas e validadas do SSQ, para uso na pratica clínica, mas não foi essa a proposta deste estudo ${ }^{(18)}$.

A avaliação da confiabilidade revelou alta consistência interna e boa confiabilidade nos questionários SSQ - Base e SSQ - B e C. O coeficiente Alfa de Cronbach foi de 0,94 para o SSQ Base e 0,99 para o SSQ B e C. Resultado também obtido nos 49 itens do SSQ nos estudos ${ }^{(7)}$, em 2015, que produziram um Alfa de Cronbach do questionário SSQ de 0,94, e em um estudo mais recente que produziram um Alfa de Cronbach de 0,93 indicando elevada consistência interna e boa confiabilidade em cada um dos domínios ${ }^{(19)}$.

Os indivíduos da amostra relataram que o questionário descreve às suas necessidades, reunindo os requisitos para ser uma valiosa ferramenta no processo de reabilitação auditiva, apesar de algumas limitações nas questões, como: ser muito extenso para a população idosa e algumas questões serem muito longas, o que leva a uma dificuldade maior na sua compreensão e interpretação.

Propõe-se a continuidade de estudo, com essa versão do SSQ adaptado culturalmente para o Português Brasileiro, selecionando amostras com número maior de participantes e o uso clínico com versões encurtadas ${ }^{(20)}$.

\section{CONCLUSÃO}

Face aos resultados obtidos, pudemos concluir que:

- O SSQ mostrou ser um instrumento de fácil aplicação que permite avaliação do benefício em indivíduos que se encontram em processo de reabilitação auditiva;

- Trata-se de um instrumento com alta confiabilidade que pode ser aplicado a indivíduos que utilizam diferentes tipos de próteses auditivas.

\section{REFERÊNCIAS}

1. WHO: World Health Organization. Deafness and hearing loss [Internet]. Geneva: WHO [citado em $2017 \mathrm{Fev}$ 17]. Disponível em: http://www.who. int/mediacentre/factsheets/fs300/en/

2. WHO: World Health Organization. International Classification of Functioning, Disability and Health: ICF. Geneva: WHO; 2001.

3. Amorim RMC, Almeida K. Estudo do benefício e da aclimatização em novos usuários de próteses auditivas. Pró-fono Rev. Atual Cient. 2007;19(1):3948. http://dx.doi.org/10.1590/S0104-56872007000100005.
4. Noble W, Ter-Horst K, Byrne D. Disabilities and handicaps associated with impaired auditory localization. J Am Acad Audiol. 1995;6(2):129-40. PMid:7772782.

5. Gatehouse S, Noble W. The Speech, Spatial and Qualities of Hearing Scale(SSQ) Int JAudiol. 2004;43(2):85-99. http://dx.doi.org/10.1080/14992020400050014. PMid:15035561.

6. Jensen N, Akeroyd M, Noble W, Naylor G. English SSQ/SSQ-B/SSQ-C v5.6 (merged pdf) [Internet]. 2016 [citado em 2017 Jan 11]. Disponível em: https://www.researchgate.net/publication/311627649

7. Gonsalez ECM, Almeida K. Adaptação cultural do questionário Speech, Spatial and Qualities of Hearing Scale (SSQ) para o Português Brasileiro. Audiol Commun Res. 2015;20(3):215-24. http://dx.doi.org/10.1590/S231764312015000300001572 .

8. Dillon H. NAL-NL1: a new prescriptive fitting procedure for nonlinear hearing aids. Hear J. 1999;52(4):10-6. http://dx.doi.org/10.1097/00025572199904000-00002.

9. Seewald R, Moodie S, Scollie S, Bagatto M. The DSL method for pediatric hearing instrument fitting: historical perspective and current issues. Trends Amplif. 2005;9(4):145-57. http://dx.doi.org/10.1177/108471380500900402. PMid:16424944.

10. Keidser G, Dillon HR, Flax M, Ching T, Brewer S. The NAL-NL2 prescription procedure. Audiology Res. 2011;1(1): e24. http://dx.doi. org/10.4081/audiores.2011.e24. PMid:26557309.

11. Holube I, Fredelake S, Vlaming M, Kollmeier B. Development and analysis of an International Speech Test Signal (ISTS). Int J Audiol. 2010;49(12):891903. http://dx.doi.org/10.3109/14992027.2010.506889. PMid:21070124.

12. ANSI: American National Standards Institute. Methods for calculation of speech intelligibility index. Washington: ANSI; 1997.

13. Singh G, Kathleen Pichora-Fuller M. Older adults' performance on the speech, spatial, and qualities of hearing scale (ssq): test-retest reliability and a comparison of interview and self-administration methods. Int J Audiol. 2010;49(10):733-40. http://dx.doi.org/10.3109/14992027.2010.491097. PMid:20874047.

14. Noble W, Gatehouse S. Interaural asymmetry of hearing loss, Speech, Spatial and Qualities of Hearing Scale (SSQ) disabilities, and handicap. Int JAudiol. 2004;43(2):100-14. http://dx.doi.org/10.1080/14992020400050015. PMid: 15035562.

15. Banh J, Singh G, Pichora-fuller MK. Age affects responses on the Speech, Spatial, and Qualities of Hearing Scale (SSQ) by adults with minimal audiometric loss. J Am Acad Audiol. 2012;23(2):81-91, quiz 139-40. http:// dx.doi.org/10.3766/jaaa.23.2.2. PMid:22353676.

16. Noble W, Gatehouse S. Effects of bilateral versus unilateral hearing aid fitting on abilities measured by Speech, Spatial and Qualities of Hearing Scale (SSQ). Int J Audiol. 2006;45(3):172-81. http://dx.doi. org/10.1080/14992020500376933. PMid:16579492.

17. Akeroyd MA, Guy FH, Harrison DL, Suller SL. A factor analysis of the Speech, Spatial and Qualities of Hearing Questionnaire (SSQ). Int J Audiol. 2011;51:262.

18. Moulin A, Richard C. Sources of variability of Speech, Spatial, and Qualities of Hearing Scale (SSQ) scores in normal-hearing and hearing-impaired populations. Int J Audiol. 2016;55(2):101-9. http://dx.doi.org/10.3109/14 992027.2015.1104734. PMid:26624277.

19. Aguiar R, Almeida K, Gonsalez E. Test-Retest Reliability of the Speech, Spatial and Qualities of Hearing Scale (SSQ) in Brazilian Portuguese. Int. Arch. Otorhinolaryngol. 23(4):380-3. https://doi.org/10.1055/s-0039-1677754.

20. Moulin A, Vergne J, Gallego S, Micheyl C. A New Speech, Spatial, and Qualities of Hearing Scale Short-Form: Factor, Cluster, and Comparative Analyses. Ear Hear. 2019;40(4):938-50. http://dx.doi.org/10.1097/ AUD.0000000000000675. PMid:30461444.

\section{Contribuição dos autores}

PTMP elaboração da pesquisa, concepção e delineamento do estudo, análise e interpretação dos dados, redação do artigo, submissão e trâmites; KA elaboração da pesquisa, análise e interpretação dos dados, concepção e delineamento do estudo, submissão e trâmites, correção da redação do artigo, aprovação da versão final. 